\title{
Reconstruction of Sun-as-Star Magnetic Field Structure and Evolution Using Filament Observations
}

\author{
Dmitri I. Ponyavin \\ Institute of Physics, St. Petersburg University, 198904, Russia \\ E-mail: ponyavin@snoopy.phys.spbu.ru
}

\begin{abstract}
A technique is used to restore the magnetic field of the Sun viewed as star from the filament distribution seen on $\mathrm{H}_{\alpha}$ photographs. For this purpose synoptic charts of the large-scale magnetic field reconstructed by the McIntosh method have been compared with the Sun-asstar solar magnetic field observed at Stanford. We have established a close association between the Sun-as-star magnetic field and the mean magnetic field inferred from synoptic magnetic field maps. A filtering technique was applied to find correlations between the Sun-as-star and large-scale magnetic field distributions during the course of a solar cycle. The correlations found were then used to restore the Sun-as-star magnetic field and its evolution in the late 1950s and 1960s, when such measurements of the field were not being made. A stackplot display of the inferred data reveals large-scale magnetic field organization and evolution. Patterns of the Sun-as-star magnetic field during solar cycle 19 were obtained. The proposed technique can be useful for studying the solar magnetic field structure and evolution during times with no direct observations.
\end{abstract}

\section{Introduction}

$\mathrm{H}_{\alpha}$ synoptic charts were first developed by McIntosh to infer magnetic fields from solar images obtained in the $\lambda 6553$ line of neutral hydrogen $\left(\mathrm{H}_{\alpha}\right.$ filtergrams). Each chart is a map of the entire solar globe in cylindrical Carrington coordinates corresponding to the mean solar rotation rate period of 27.2753 days. $\mathrm{H}_{\alpha}$ charts show patterns of neutral lines (lines of magnetic polarity reversals) mapped from filaments and a variety of chromospheric features governed by magnetic fields. Correlations of $\mathrm{H}_{\alpha}$ synoptic charts with direct large-scale magnetic field measurements, like the Stanford data, were well established.

Thus, the inference of lines of polarity inversion could serve as a valuable alternative to data derived by magnetograph observations, especially in the absence of direct measurements. McIntosh (1979) published an Annotated Atlas of $\mathrm{H}_{\alpha}$ synoptic charts for solar cycle 20. Furthermore, Makarov and Sivaraman (1985) inferred photospheric magnetic fields from $\mathrm{H}_{\alpha}$ filtergrams back to the beginning of the century. The purpose of our paper is to show a possibility to infer from $\mathrm{H}_{\alpha}$ synoptic charts another important feature of solar magnetism, the Sun-as-Star magnetic field. 


\section{Data Analysis and Results}

Severny (1969) was first to discover that the Sun was a magnetic variable star. To prove this, he used the mean magnetic field observations seen as a star provided in Crimea since 1968. Later close associations between the Sun-as-Star magnetic field and interplanetary magnetic field sector structure of the solar wind were established by Scherrer et al. (1977). Kotov et al. (1977) showed that the day-today Sun-as-Star magnetic field measurements are highly correlated (0.87) with the large-scale magnetic field inferred from $\mathrm{H}_{\alpha}$ observations. The procedure they used was based on averaging the $\mathrm{H}_{\alpha}$ synoptic charts over a range of $50^{\circ}$ in latitude. By changing the longitude window, they found the best correlation with 5 days, corresponding approximately to $70^{\circ}$ in longitude.

A similar averaging procedure was applied in this paper. $\mathrm{H}_{\alpha}$ synoptic charts were transformed into digital form by scanning latitudes of intersection of neutral lines with the meridian of Carrington longitude passage. The grid used for interpolation contained 36 longitude bins, each 10 heliographic degree wide, and 18 latitude bins, each representing an equal increment of $10^{\circ}$. Magnetic fields were specified to be +1 and -1 to describe the positive and negative polarity. Values in the vicinity of neutral lines were then linearly interpolated.

A moving window of latitudinal and longitudinal extents of $60^{\circ}$ by $60^{\circ}$ was selected corresponding to the maximum correlation with the magnetograph data. To decrease noise an additional smoothing technique was applied: 5 running synoptic charts were superposed to improve the reconstruction results. The main results of this smoothing procedure are the following:

- Uncertainties in inferring neutral lines using a single $\mathrm{H}_{\alpha}$ synoptic chart could be reduced. It is more important for the much noisier $\mathrm{H}_{\alpha}$ data in the Makarov and Sivaraman reconstruction in the absence of magnetograph observations.

- Long-lived features in the large-scale magnetic field organization (active longitudes and latitudes) are more pronounced.

- Effective synoptic charts of magnetic fields reveal not only the polarity distribution but also effective fluxes of the magnetic field.

- Flux balances in the positive and negative polarity are also supported.

- The contribution of the axisymmetrical dipole component that is poorly manifested in the measured line-of-sight magnetic field has been increased.

Full-disk measurements of the mean magnetic field began at Stanford in 1975 and have continued through the present. A summary of Stanford day-today observations of the mean magnetic field seen as star is presented in Figure 1. A running filtering technique was applied to cover gaps and reveal the large-scale magnetic field organization. Figure 1 shows a Bartels display of available data covering solar cycles 21 and 22 . Time increases to the right in the panel. In this format horizontal patterns indicate long-lived structures that return with a 27-day synodic rotational period. Sunspot cycle activity during the period 19761996 are shown at the top. Similar patterns of the mean magnetic field restored using our technique for solar cycles 19 and 20 have been presented in Figures 2 
and 3, respectively. A large-scale organization of magnetic fields can clearly be seen from the Figures. Original magnetic fields reach their maximum values near solar maximum, whereas amplitude variations of the inferred field are less pronounced over the solar cycle. However, comparison of solar cycles 21 and 22 with the previous cycles indicates the general character of the magnetic polarity changing through the whole period of observations. Two systems of magnetic field rotation rate could be observed. The first pattern indicates a rotation rate with a period greater, and the second a little slower than 27 days. These periods are similar to those found earlier by Kotov (1986), 28.2 and 26.9 days, that may reflect the north-south asymmetry of solar activity (Antonucci et al. 1990). In summary, the technique presented here can serve as a meaningful tool to probe the large-scale organization of solar magnetic fields and its evolution in the past.

\section{References}

Antonucci, E., Hoeksema, J.T. and Scherrer P.H. 1990, ApJ, 350, 296

Kotov, V.A., Stepanyan, N.N. and Scherbakova, Z.A. 1977, Izv. KrAO, 66, 75 Kotov, V.A. 1986, Izv. KrAO 75, 39

Makarov, V.I. and Sivaraman, K.R. 1986, Atlas of $\mathrm{H}_{\alpha}$ synoptic charts for solar cycle 19 (1955-1964), Kodaikanal Obs. Bull. 7, 2-138

McIntosh P.S. 1979, Annotated atlas of $\mathrm{H}_{\alpha}$ synoptic charts for solar cycle 20 (1964-1974), Report UAG-70, WDC-A, Boulder

Scherrer, P.H. et al. 1977, Solar Phys., 52, 3

Severny, A. 1969, Nature 224, 53
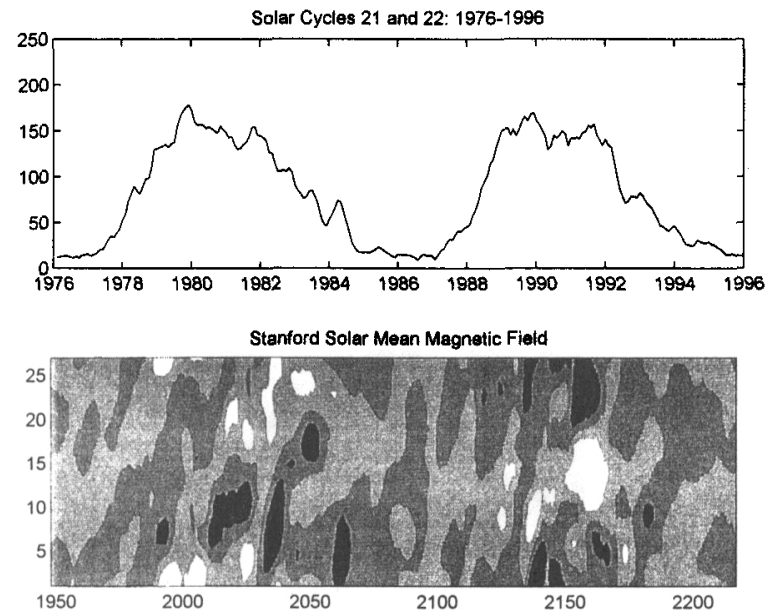

Figure 1. Bartels display of the mean magnetic field measured at Stanford. Bottom: time increases to the right for Bartels rotations 1948-2217. Negative polarity is darker. Horizontal patterns indicate long-lived structures with 27-day synodic rotation periods. Top: monthly mean sunspot numbers smoothed during 1976-1996. 

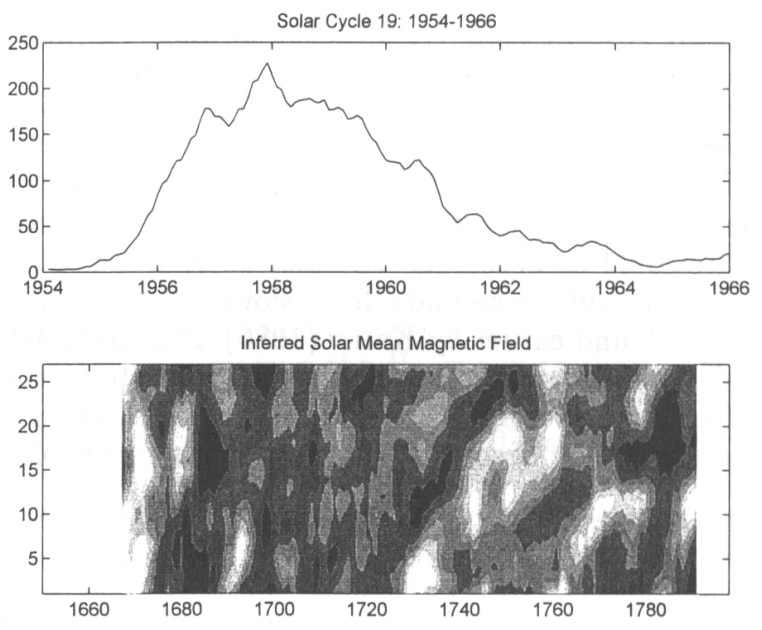

Figure 2. Bartels display of inferred mean magnetic field from $\mathrm{H}_{\alpha}$ synoptic charts. Same as Figure 1 but for Bartels rotations 1650-1798.
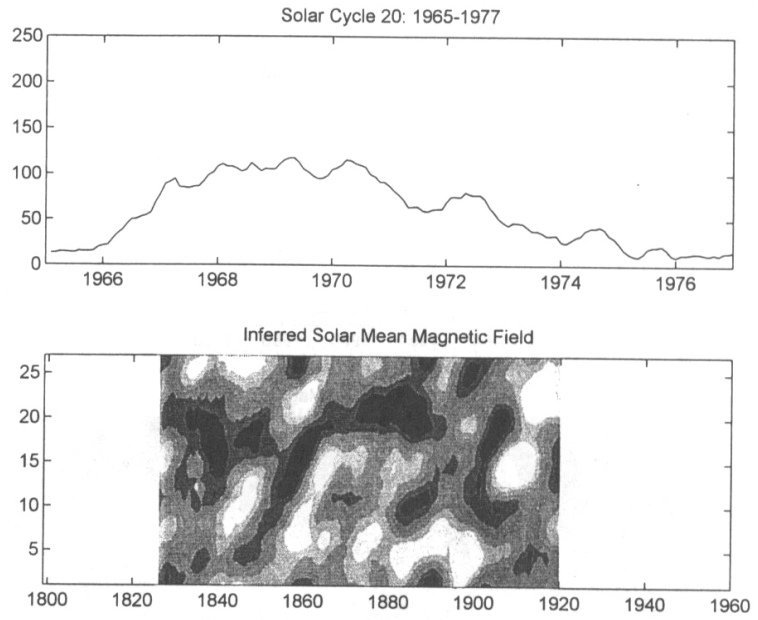

Figure 3. Same as Figure 1 but for Bartels rotations 1820-1920. 